\title{
WikiJournal of Medicine, the first Wikipedia-integrated academic journal
}

Thomas Shafee, ${ }^{1}$ Diptanshu Das, ${ }^{1}$ Gwinyai Masukume, ${ }^{2}$ and Mikael Häggström ${ }^{3 *}$

\begin{abstract}
WikiJournal of Medicine is an open access, peer reviewed journal free of publication charges for its authors. It publishes both research and reviews. It was created in 2014 and has grown rapidly since then. This editorial will highlight its unique features and the developments seen in 2016.
\end{abstract}

\section{Introduction}

WikiJournal of Medicine was established in 2014 as a bridge between the Wikipedia community and medical academia. In the academic community there is a strong and growing movement towards open access journal models, and open access journals already make up 12\% of all sciences and humanities journals. ${ }^{[1]}$ Some have also experimented with publication to Wikipedia. ${ }^{[2][3]}$ Wikipedia remains the world's largest encyclopedia at 5.3 million articles in English, ${ }_{1}^{[4]}$ and is amongst the most read online sources of medical information ${ }^{[5]}$ Wikipedia has a quality assessment system whereby a page can be reviewed by 4-10 independent editors to determine its suitability to be promoted to a 'featured article' (4900 articles). ${ }^{[6]}$ However, there remain many science and medicine pages in need of improvement. ${ }^{[7]}$ Improved integration between academic journals and the encyclopedia stands to benefit both systems. ${ }^{[8]}$

The WikiJournal of Medicine combines scholarly quality control with wiki editing. It runs on a wiki hosted by the Wikimedia Foundation (WMF), with minimal requirements for technical maintenance by journal participants. It can therefore publish free of charge for both authors and readers, using the uncommon "diamond

\footnotetext{
${ }^{1}$ Editorial board, WikiJournal of Medicine

${ }^{2}$ Associate editor in chief, WikiJournal of Medicine

${ }^{3}$ Editor in chief, WikiJournal of Medicine

*Author correspondence: editor.in.chief@wijoumed.org

ORCID1: 0000-0002-2298-7593

ORCID2: 0000-0002-7221-5022

ORCID3: 0000-0002-9251-0264

ORCID4: 0000-0002-2732-7631

Supplementary material: Table $\mathrm{S}_{1}$

Licensed under: CC-BY-SA

Published 15-01-2017
}

open access" model. ${ }^{[1][9]}$ It is also editable by anyone, allowing a large group of casual associate editors to aid in formatting and copyediting publications, supporting the main editorial board. It also has the flexibility and transparency to support both public peer review (with reviewers choosing to either contribute under their own name, or anonymously ${ }^{[10]}$ and post-publication peer review. ${ }^{[11]}$ Articles so far published in WikiJournal of Medicine have been incorporated into Wikipedia articles, which collectively exceed 20 million pageviews during 2016, allowing an enormous reach for a journal of its age (supplementary table). Finally, this year it became the second journal to put an existing Wikipedia article through academic peer review. ${ }^{[12][3]}$ The Cerebellum article was published in June and the peer-reviewed version replaced the previous Cerebellum Wikipedia page. ${ }^{[12]}$

\section{Developments in 2016}

In 2016 the editorial board grew to 10 members, with the addition of: $G$ Vandegrift, D Das, T Shafee and M Laurent. The editorial board oversaw key improvements of the journal's organisational structure. Formal bylaws have been adopted, improving the journal's compliance with the International Committee of Medical Journal Editors recommendations. It has also been accepted into the Directory of Open Access Journals and has been registered as a non-profit organisation. Approval for the formation of a Wikimedia user group in June facilitated this process, and paved the way for the journal being granted funding by the WMF through 2017. Furthermore, this year many new participants have joined as associate editors and listed peer reviewers to help the smooth running of the journal. 


\section{A new look}

The functional changes have been complemented by updates of the structure and formatting of the journal's website as well as the published articles. The new design improves the readability of the articles as PDFs and online ${ }^{[13]}$ whilst retaining the advantages of the MediaWiki backbone used by the website. ${ }^{[14]}$ The journal's name was voted upon by the community, with WikiJournal of Medicine replacing the former name Wikiversity Journal of Medicine.

\section{Expansion of outreach}

The WikiJournal of Medicine is increasing in notability, through multiple efforts by the journal's editorial board. The journal's model and features were presented at the conferences Wikimania, WikiConference India and OpenCon Ranchi during 2016. These presentations showcased the journal to people working with various WMF projects or Open Access initiatives in an attempt to further unify academics with the community of Wikipedians. Additionally, interest by the medical community and general public have lead to our activities being highlighted in external publications in the popular press. ${ }^{[15][16][17]}$

\section{New WikiJournals}

The editorial board believes that the WikiJournal model is a promising format for other fields, and new WikiJournals are already under development. This year has seen the creation of a formal Wikimedia user group that can help to organise the efforts of new journals, and provide a unified naming and logo series. The scope of WikiJournals is also extending, with the development of WikiJournal of Science and interest in the creation of a humanities journal as well as a similar science journal in French.

The WikiJournal of Science's focus on existing Wikipedia pages inspired us to extend from the WikiJournal of Medicine's original remit of adding new information to Wikipedia, to also include scholarly review of existing information. As a consequence, the quality of existing articles is upgraded through the academic peer review process. Meanwhile, Wikipedia authors can obtain credit for their work developing Wikipedia entries, and can be cited by external works just as in other scholarly publications. We therefore hope that this publication will act as a flagship WikiJournal with the format being extended to other fields.

\section{The year ahead}

One of the greatest challenges for a non-profit, volunteer-run journal is promoting the publication. ${ }^{[18]}$ In a crowded academic publishing landscape with an increasing number of predatory journals, it is increasingly important for new journals to justify their existence and highlight their legitimacy ${ }^{[19][20]} \mathrm{A}$ major aim for 2017 is therefore to publicise, amongst medical and research professionals, this journal's potential to bring medically important material to the vast readership of Wikipedia with no charge for authors. To do this, the WikiJournal of Medicine needs to highlight its unique and novel format. ${ }^{[21]}$

Through 2017, the editorial board plans to implement extensive outreach, both to the Wikipedia community and to the medical community in general. Firstly, the journal will be engaging Wikipedia's medical community and other editor groups, as well as the WMF's outreach and education initiatives. The board will also continue to increase international awareness at medical schools and research institutes. Additionally, a grant from the WMF will be used to fund a new WikiJournal of Medicine "Best article Prize" to be awarded to authors of articles of exceptional scientific content, readability and value to the general public, as voted by an external judging panel. Articles of any format submitted by September 2017 are eligible for a competition with prizes of US $\$ 200, \$ 100$, and $\$ 50$. This continued outreach should bring the publication rate up for the journal to be eligible for MEDLINE/PubMed indexing in 2017. Inclusion in these databases will in turn increase the credibility of the journal, and attract further article submissions. The high exposure that WikiJournal of Medicine articles have through Wikipedia will likely lead to good citation rates.

We therefore expect the year ahead to be positive for the journal. The medical and research communities are increasingly aware of the need for improved content on Wikipedia, and this journal is well positioned to facilitate that process.

\section{References}

1. Christian Fuchs, Marisol Sandoval (2013). "The diamond model of open access publishing: why policy makers, scholars, universities, libraries, labour unions and the publishing world need to take non-commercial, nonprofit open access serious". tripleC 11 (2).

2. Wodak, Shoshana J.; Mietchen, Daniel; Collings, Andrew M.; Russell, Robert B.; Bourne, Philip E. (2012). "Topic Pages: PLoS Computational Biology Meets Wikipedia". PLoS Computational Biology 8 (3): e1002446. doi:10.1371/journal.pcbi.1002446. ISSN 1553-7358.

3. Heilman, JM; De Wolff, J; Beards, GM; Basden, BJ (2014). "Dengue fever: a Wikipedia clinical review". Open Med 8 (4): e105-15. PMID 25426178. PMC 4242787.

4. "Wikipedia Statistics (All languages)". stats.wikimedia.org. Retrieved 2017-01-14. 
5. Laurent, M. R.; Vickers, T. J. (2009). "Seeking Health Information Online: Does Wikipedia Matter?". Journal of the American Medical Informatics Association 16 (4): 471-479. doi:10.1197/jamia.M3059. ISSN 1067-5027.

6. Lipka, Nedim; Stein, Benno (2010). Identifying featured articles in wikipedia. pp. 1147. doi:10.1145/1772690.1772847.

7. Volsky, Peter G.; Baldassari, Cristina M.; Mushti, Sirisha; Derkay, Craig S. (September 2012). "Quality of Internet information in pediatric otolaryngology: A comparison of three most referenced websites". International Journal of Pediatric Otorhinolaryngology 76 (9): 1312-1316. doi:10.1016/j.ijporl.2012.05.026. PMID 22770592.

8. Masukume, Gwinyai; Kipersztok, Lisa; Das, Diptanshu; Shafee, Thomas M

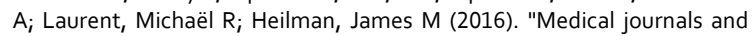
Wikipedia: a global health matter". The Lancet Global Health 4 (11): e791. doi:10.1016/S2214-109X(16)30254-6. ISSN 2214109X.

9. Frank, Martin (2013). "Open but Not Free - Publishing in the 21st Century". New England Journal of Medicine 368 (9): 787-789. doi:10.1056/NEJMp1211259. ISSN 0028-4793.

10. van Rooyen, Susan; Godlee, Fiona; Evans, Stephen; Black, Nick; Smith, Richard (1999). "Effect of open peer review on quality of reviews and on reviewers' recommendations: a randomised trial". BMJ 318 (7175): 23-27. doi:10.1136/bmj.318.7175.23. ISSN 0959-8138.

11. Winker, Margaret (2015). "The promise of post-publication peer review: how do we get there from here?". Learned Publishing 28 (2): 143-145. doi:10.1087/20150209. ISSN 09531513.
12. Wright, Marion; Skaggs, William; Årup Nielsen, Finn (2016). "The Cerebellum". WikiJournal of Medicine 3 (1). doi:10.15347/wjm/2016.001. ISSN 20024436.

13. Dyson, Mary C. (2004). "How physical text layout affects reading from screen". Behaviour \& Information Technology 23 (6): 377-393. doi:10.1080/01449290410001715714. ISSN 0144-929X.

14. Barrett, Daniel J. (2008-10-14) (in en). MediaWiki. "O'Reilly Media, Inc.". ISBN 9780596156541.

15. Gwinyai Masukume, James Heilman, Mikael Häggström (2016-05-24). "Why getting medical information from Wikipedia isn't always a bad idea". The Conversation.

16. Thomas Shafee (2016-10-18). "Wikipedia is already the world's 'Dr Google' - it's time for doctors and researchers to make it better". The Conversation.

17. "Doctors call on medical journals to improve health content on Wikipedia". Medical News Today. 2016-10-19.

18. "How To Promote an Open Access Journal". Open Science. 2013-01-16.

19. McLeod, Alexander; Savage, Arline; Simkin, Mark G. (2016). "The Ethics of Predatory Journals". Journal of Business Ethics. doi:10.1007/s10551-0163419-9. ISSN 0167-4544.

20. Clark, J.; Smith, R. (2015). "Firm action needed on predatory journals". BMJ 350 (jan16 1): h210-h210. doi:10.1136/bmj.h210. ISSN 1756-1833.

21. Swan, Alma; Brown, Sheridan (2004). "Authors and open access publishing". Learned Publishing 17 (3): 219-224. doi:10.1087/095315104323159649. ISSN 09531513. 